\title{
La rumeur du cachet au Burundi (1960-1961)
}

Essai d'interprétation d'une conversation nationale sur le politique

\section{Christine Deslaurier}

\section{(2) OpenEdition}

\section{Journals}

Édition électronique

URL : http://journals.openedition.org/etudesafricaines/5466

DOI : 10.4000 /etudesafricaines. 5466

ISSN : 1777-5353

Éditeur

Éditions de l'EHESS

\section{Édition imprimée}

Date de publication : 25 juin 2005

Pagination : $545-572$

ISBN : 978-2-7132-2048-7

ISSN : 0008-0055

\section{Référence électronique}

Christine Deslaurier, «La rumeur du cachet au Burundi (1960-1961) », Cahiers d'études africaines [En ligne], 178 | 2005, mis en ligne le 30 juin 2008, consulté le 02 mai 2019. URL : http://

journals.openedition.org/etudesafricaines/5466 ; DOI : 10.4000/etudesafricaines.5466

Ce document a été généré automatiquement le 2 mai 2019.

(c) Cahiers d'Études africaines 


\title{
La rumeur du cachet au Burundi (1960-1961)
}

\author{
Essai d'interprétation d'une conversation nationale sur le politique
}

Christine Deslaurier

1 Au royaume du Burundi, alors encore sous tutelle belge ${ }^{1}$, le début des années 1960 a été marqué par une forte effervescence politique, liée aux réformes hâtivement engagées par l'administration coloniale en vue de l'indépendance (Deslaurier 1998, 2002). À partir de la fin 1959, dans la perspective des échéances électorales annoncées par les nouveaux textes législatifs, de nombreux partis politiques ont été créés ${ }^{2}$. Leurs activités de propagande ont emprunté aux canaux usuels de la communication politique, écrite et orale: journaux, tracts, discours et chansons ont envahi les collines ou résonné dans les cabarets et les meetings. Grâce aux archives conservées à Bruxelles et à Bujumbura et aux témoignages recueillis au Burundi sur cette période, on connaît désormais assez bien la teneur des messages diffusés par les partis et leurs militants, leur mode de diffusion et leur impact global sur la population d'alors, essentiellement rurale et analphabète ${ }^{3}$. Un constat se dégage d'emblée : quel que soit le support utilisé pour délivrer l'information politique, in fine c'est toujours par la transmission orale que les nouvelles ont circulé en milieu rural ou dans les centres urbains. Or parmi les différentes formes et voies empruntées par l'oralité dans la médiatisation des informations, les rumeurs (urukurukuru, ibihuha ${ }^{4}$ ) ont tenu une place tout à fait considérable, voire prépondérante. Constituant un genre de conversation à part entière, comme un polylogue local, régional ou même national, elles ont proliféré dans la forte densité des relations sociales en concurrençant sans peine les médias modernes embryonnaires ${ }^{5}$. Leur cycle de vie, comme celui des tracts dans le domaine de l'écrit, a épousé la chronologie et l'amplitude des saccades de la transition administrative et politique (Deslaurier 2002: 668-669). Par un processus de recyclage et de réactualisation de récits mythiques, elles ont irrigué une culture populaire déjà riche d'images et de représentations spécifiques du pouvoir, et l'ont mise au contact des figures nouvelles de la "modernité " politique portées par les réformes. À plusieurs titres, ces rumeurs permettent donc de connaitre l'histoire 
politique du Burundi et l'on saurait d'autant moins s'en passer que les sources sont disponibles pour retracer leurs grandes lignes, leur contenu et leur influence.

On ne perçoit souvent qu'un écho assourdi de l'immense brouhaha déclenché par le débat politique dans les sphères populaires africaines, qui nous parvient au travers des témoignages oraux. Mais dans le cas des rumeurs qui caractérisent précisément certaines ambiances politiques et en traduisent aussi très bien les perceptions, on dispose pour le Burundi d'une documentation écrite importante, complémentaire des sources orales. En particulier les agents de la Sûreté du Ruanda-Urundi, au service d'une colonisation qui s'était avisée très tôt de connaître "l'état d'esprit des populations", ont consigné de manière détaillée et réitérée les bruits et les rumeurs qui circulaient sur les collines ou dans les cités indigènes (Deslaurier 1998 : 63-64). Les bulletins secrets qu'ils ont rédigés à ce propos en 1960 et 1961, et d'autres documents administratifs, permettent d'établir une recension assez précise de ces rumeurs et d'en évaluer plutôt correctement l'intensité et l'influence. Toutefois la matière abonde, et l'on risquerait de morceler la restitution de l'atmosphère saisissante de la transition en étudiant des bruits multiples qui n'informent pas de manière continue le champ de l'interprétation politique. Aussi cet article proposet-il l'analyse d'une rumeur récurrente des années 1960 et 1961, qui constitue un cas particulièrement intéressant. La "rumeur du cachet », par sa durée chronologique, son extension géographique et ses retombées concrètes, forme en effet un exemple idéal pour éclairer les tensions et les enjeux politiques de la décolonisation burundaise car on y appréhende à la fois les modes de structuration du débat public, les représentations populaires du pouvoir et les diverses facettes de l'imaginaire collectif convoquées pour dire la nouvelle politique.

3 La démarche adoptée pour conduire cette étude s'inspire des nombreux travaux consacrés aux rumeurs dans le champ des sciences humaines et sociales (histoire, anthropologie, sociologie, sciences de la communication...), qui se sont beaucoup renouvelés ces dernières années. À l'origine, les recherches ont situé le phénomène de la rumeur dans un registre antagonique, en construisant un concept et des dispositifs pour l'étudier fondés sur les dualismes du vrai et du faux, de l'officiel et de l'officieux, ou encore du rationnel et de l'irrationnel (Reumaux 1996:110,149), et cette posture héritée des hypothèses positivistes $\mathrm{du}$ début $\mathrm{du} \mathrm{xx}^{\mathrm{e}}$ siècle a pendant longtemps dirigé le questionnement des chercheurs qui ont centré leur intérêt sur la psychologie du témoignage et porté attention surtout au mode de transmission de la rumeur et aux distorsions de l'information lors de sa réplication (Froissart $2000: 183-190)$. Toutefois, la rupture avec ces perspectives strictement fonctionnelles et déterministes est maintenant consommée, et les réflexions sur le sens et les dynamiques de la rumeur s'organisent différemment depuis qu'elle est entrevue moins comme un "accident de la communication " que comme l'une de ses formes dans laquelle s'énonce autrement l'information (Reumaux 1996). Ainsi, certains l'envisagent comme une construction discursive sur le pouvoir et insistent sur ses propriétés spécifiques comme mode informel de contradiction des instances étatiques et des autorités publiques: niché dans les interstices de l'oralité laissés vacants par les circuits d'information officiels, il s'agirait d'un récit de doléances et de résistance des plus faibles (Scott 1985), ou d'une narration collective témoignant d'une pluralisation du débat public (Morin 1982). D'autres considèrent que loin d'être la marque d'une cohésion communautaire forte, la rumeur est en réalité le signe de sa fragilité, traduisant une intense compétition politique entre élites et opérant comme une mesure répétée des insertions et des légitimités dans les groupes 
sociaux (Gluckman 1963). Ailleurs, dégagée des carcans du rationalisme et de l'authentification des faits, la compréhension des dimensions métaphoriques ou légendaires de la rumeur a été libérée quand on a pris « au sérieux » son récit pour en extraire la substance imaginaire et les ressorts sociaux et politiques majeurs (White 2000a, 2000b). Ces différentes approches, de même que celle qui traite la rumeur comme une manipulation délibérée de l'opinion, balisent largement le territoire que cet article entend reconnaître, mais ses frontières n'en sont pas pour autant figées : dans la mesure où cette rumeur s'ancre dans une culture historique spécifique et figure un monde politique particulier, on doit aussi prêter attention aux conditions locales de son épanouissement et de son extension.

La rumeur du cachet (1960-1961)

4 Répétons-le, les sources disponibles pour travailler sur les rumeurs dans le Burundi de la décolonisation sont nombreuses, et si l'on peut étudier plus précisément la rumeur du cachet, c'est parce qu'à ce sujet elles sont encore plus complètes et diversifiées. D'abord une variété de documents écrits a été retrouvée dans les archives belges et burundaises, des dépêches de presse aux rapports administratifs et politiques émanant des responsables belges ou des autorités "indigènes », en passant par des procès-verbaux d'enquête policière ou par les fameux bulletins des services de la Sûreté évoqués plus haut. Ensuite, on peut conjuguer l'apport de ces écrits avec une série d'entretiens réalisés sur le terrain auprès de témoins de l'époque. Ces enquêtes ont été menées dans différentes régions du pays en 1990, par un étudiant burundais (Bukuru 1990) et par moimême dans le cadre de nos travaux respectifs consacrés au développement politique burundais dans les années $1960^{6}$. Par ailleurs, à l'occasion d'un récent séjour au Burundi, j'ai réalisé de nouveaux entretiens avec des observateurs ayant assisté à l'incident le plus grave lié à la rumeur du cachet, qui eut lieu en juin $1961^{7}$. Analysées conjointement et recoupées, les informations fournies par cet ensemble de sources permettent d'écrire le texte de base de cette rumeur et aident à établir une chronologie de sa circulation, en opérant une distinction entre les différentes phases de sa diffusion, dont certaines ont eu des retentissements considérables, à la fin 1960 et au milieu 1961. Elles éclairent aussi les éléments fixes de la rumeur, répercutés dans chacune de ses versions, et soulignent l'importance des motifs mythiques et des images quasi-subliminales qui lui ont conféré sa puissance.

Textes de la rumeur, contextes de sa diffusion

5 Les premières traces d'une rumeur centrée autour d'un cachet sont apparues en novembre 1960 dans le territoire de Ngozi, situé au Nord du Burundi, lors des scrutins locaux qui devaient aboutir à la constitution des premiers conseils communaux. Dans plusieurs communes méridionales de Ngozi, l'agent de la Sûreté chargé de la surveillance des élections nota un "refus général de voter dès le début des opérations » que les personnes interrogées à ce moment justifièrent par les risques encourus à participer à la consultation (BUR 61/2: 28 novembre 1960). Une information circulait en effet selon laquelle « il arriver[ait] malheur à ceux qui aur[aient] dans leur carnet d'identité le cachet Yatoye » (ibid.) - ce dernier attestant du passage devant les urnes ${ }^{8}$. Parfois plus précise, la menace suggérait encore que « ceux qui voteraient seraient marqués et n'auraient qu'à partir quand les Belges partiraient » (BUR 75/5 : 10 avril 1961), une perspective qui prenait sens au moment où l'on évoquait de plus en plus l'accession à l'indépendance du Burundi. De fait, cette rumeur provoqua dans plusieurs communes du territoire de Ngozi la défection d'une bonne partie des électeurs ou leur abstention presque totale. Ainsi dans 
les communes de Mubogora et de Gaheta qui regroupaient plus de 6000 électeurs potentiels, à peine 2 à $3 \%$ des inscrits prirent part au scrutin et dans celles, limitrophes, de Rango et Butaganzwa (plus de 8000 inscrits en tout), le plafond participatif demeura bien en dessous de ce qu'il fut ailleurs dans la région ou dans le pays ${ }^{9}$. D'autres éléments peuvent être invoqués pour expliquer les réticences au vote dans ces communes, à commencer par les positions locales du principal parti d'opposition à l'administration belge, l'UPRONA (Unité et progrès national), qui prônait à ce moment le boycottage d'une série d'initiatives coloniales, parmi lesquelles les élections. Dans une région où, comme on le verra, les rivalités partisanes étaient exacerbées par la compétition entre quelques grandes figures de la monarchie, la rumeur du cachet n'a pas créé une nouvelle tension politique - elle était déjà vive -; elle l'a accompagnée. Cependant, le lien entre la rumeur et l'absentéisme électoral est frappant si l'on considère les accommodements qu'ont tenté d'obtenir certains électeurs en proposant « de voter à condition que l'on ne mette pas le cachet "Yatoye" ("a voté") sur leur livret d'identité, ce qui ne put évidemment être accordé en raison de la réglementation électorale " (AI 4369/4: 11 janvier 1961). En définitive, dans leurs motifs d'abstention, la crainte de l'empreinte laissée sur le papier parait avoir supplanté celle d'enfreindre la consigne de boycott édictée par les militants locaux de l'upronA. Aussi, dans cette situation, on peut dire que le refus du vote a été davantage le résultat de la diffusion de la rumeur que la preuve d'une véritable hostilité à l'encontre du scrutin local.

6 Après cette phase initiale et ses répercussions électorales, la rumeur du cachet a poursuivi son développement en 1961 et, lorsqu'elle a atteint son stade crucial, au milieu de l'année, a entraîné des conséquences tragiques. Relativement territorialisée au moment des élections communales et rattachée à celles-ci par le biais de son objet principal, le tampon Yatoye, la rumeur s'est donc étendue dans les diverses régions du pays au cours du premier semestre de l'année 1961, en prenant discrètement de l'ampleur tandis que son contenu changeait de sens et qu'il s'avérait parfois « vérifiable ». À cette époque, le pivot du texte est resté un cachet, mais celui-ci n'avait plus ni forme, ni consistance, ni mention déterminées et il n'était plus apposé sur les carnets d'identité par des fonctionnaires européens, mais appliqué sur les corps par des militants, comme une marque d'affiliation politique. Le récit rapportait que des propagandistes de formations politiques opposées à l'UPRONA, en particulier ceux du Parti démocrate chrétien (PDC) qui était le chef de file des partis favorables à une indépendance « préparée » (c'est-à-dire le maintien temporaire de la Tutelle belge sur le Burundi), marquaient la peau de leurs sympathisants pour " permettre à l'administration de les reconnaître et de les protéger " (BUR 74/4 : 28 juin 1961). Mais il s'accompagnait aussi de l'idée que celui qui serait tatoué de la sorte risquait de s'exposer, « au jour de l'indépendance, à la vindicte de l'UPRONA et du Mwami » (ibid.). Déjà l'indépendance se profilait, confirmée par la présence dans le pays de délégués onusiens plutôt bienveillants à l'égard de l'uPRONA ; aussi la menace des représailles constituait-elle un danger imminent pour les membres du PDC et des partis alliés, et plus généralement pour tous ceux sur qui pouvait être imprimé ce cachet. Or, il se trouve qu'au milieu de l'année 1961 l'avertissement se clarifia véritablement, quand des scènes publiques de « marquage » se déroulèrent dans différentes régions du pays, qui validèrent en quelque sorte la rumeur et justifièrent des attitudes de fuite et de protection parmi la population.

7 Les premiers incidents signalés eurent lieu dans la province de Makamba, au sud du pays, bien loin de l'épicentre « historique » de la rumeur de Gaheta-Mubogora. Vers la fin du 
mois de mai 1961, des échauffourées se produisirent à la suite de séances de "tatouage " menées par des individus non identifiés qui battirent en retraite devant les hommes de la région (BUR 74/4 : 28 juin 1961). Quelques semaines plus tard, à la mi-juin 1961, c'est dans la région de Rutana, à l'Est du pays, que se produisirent de telles scènes. Selon le ministre de l'Intérieur de l'époque, Jean-Baptiste Ntidendereza (par ailleurs leader du PDC), l'une d'elles se conclut par le lynchage d'un « estampilleur » présumé (ibid.). Enfin l'événement le plus sérieux eut lieu une semaine plus tard, à l'autre extrémité du pays, dans une province située non loin d'Usumbura ${ }^{10}$. L'hebdomadaire Rudipresse, organe de presse officiel de l'administration coloniale, en rapporta les détails de la manière suivante :

« Dimanche le 25 juin 1961, un grave incident a eu lieu au marché de Muzinda, en territoire de Bubanza. Un indigène attablé dans un bar sort en hurlant et produit la marque d'un cachet sur le bras qui lui aurait été apposé par des partisans du PDC qui, suivant la rumeur publique, voudraient désigner ainsi leurs ennemis politiques. Il s'agirait d'un cachet marqué "Feuer original" trempé dans de l'acide. La foule du marché, estimée à 500 personnes, se rassemble, arrête le coupable désigné par la victime, l'assomme et le fait brûler sur un bûcher construit à la hâte. Le gardien du marché et le tenancier du cabaret qui tentaient de s'interposer furent sauvagement frappés et refoulés. La police a procédé à une dizaine d'arrestations » (BUR $60 / 2: 1^{\mathrm{er}}$ juillet 1961).

8 Cette dépêche, jointe à d'autres rapports et aux témoignages établissant une version populaire du drame de Muzinda sensiblement identique, fait apparaître quelques évolutions dans le texte de la rumeur, après l'apparition des dimensions physiques du marquage au cachet signalées auparavant. On note d'abord une inversion des données initiales puisque les cibles désignées ne sont plus les pédécistes ou assimilés, qui pouvaient en 1960 profiter d'une protection coloniale ou subir des pressions de l'UPRONA en fonction d'un cachet réglementaire, mais les upronistes, pourchassés par leurs adversaires politiques ${ }^{11}$. Le cachet n'est donc plus le symbole d'une protection offerte par l'État colonial, mais bien la marque d'une agression. Cette version est confirmée par les observateurs du drame rencontrés à Muzinda dernièrement: ceux de l'uprona étaient visés dans ces attaques ${ }^{12}$. Ensuite à l'encre se substitue l'acide, comme si l'on franchissait une étape aggravante de la marque imprimée sur un document à la brûlure irrémédiable de l'épiderme, en passant du registre matériel au répertoire corporel. Enfin, quand la mention Yatoye paraissait en 1960 plutôt limpide, le cachet qui aurait été retrouvé en 1961 produit un message plus énigmatique, dans une langue étrangère. Seule la dépêche de Rudipresse signale cette mention Feuer original inscrite sur le tampon, qui peut faire sens avec la brûlure de l'acide si on la traduit depuis l'allemand par « feu original ». Mais si les premières notes des enquêteurs indiquaient bien que le cachet « sembl[ait] être d'origine allemande », elles mentionnaient aussi que les seules lettres déchiffrées étaient "fern... orig... » (BUR 75/5 : [juillet 1961]). Par ailleurs, à Muzinda, les témoins interrogés ont décrit la marque produite par l'acide et, avec beaucoup d'hésitation, la forme ronde qu'avait le cachet, mais aucun ne s'est souvenu d'un tel message inscrit sur le tampon : selon eux le cachet disait simplement qu'on était membre du PDC ${ }^{13}$. Aussi, la parabole du «feu original » pourrait bien n'être qu'une reconstruction imaginaire due au rédacteur de la dépêche de presse, une forme de rationalisation des éléments incomplets de l'enquête menée par les autorités.

9 Tous ces glissements par rapport au texte initial n'ont pas fondamentalement affecté le sens de la rumeur dont les invariants sont restés les mêmes en 1960 et 1961 : un tampon, une marque de positionnement politique, des conséquences fâcheuses. En revanche, ils 
témoignent de phénomènes d'adaptation typiques des rumeurs dont l'actualisation perpétuelle ne contredit ni la temporalité ni les occurrences locales. Le renversement des perspectives à propos des cibles potentielles des opérations de «cachetage " trahit par exemple l'évolution du contexte politique entre 1960 et 1961, comme si la rumeur s'était adaptée à la nouvelle donne des rapports de force entre les partis et l'administration de la Tutelle. Apparue à l'heure des élections communales de 1960, au moment où les campagnes d'information organisées par l'administration coloniale battaient leur plein et quand le PDC et ses alliés, appuyés par cette dernière, primaient dans le paysage politique national, la rumeur concernait au départ les membres de ces partis, ou présumés tels. Mais transformée l'année suivante lorsque l'uPRONA, désormais soutenue par une commission des Nations-Unies pour le Ruanda-Urundi et autorisée à développer ses activités de propagande, tint à son tour le haut du pavé, c'est aux partisans de ce dernier parti qu'elle s'est alors adressée. La rumeur s'arrimait ainsi aux aléas des changements d'influence des partis sans que son message ne se modifie pour autant. En effet, le premier niveau d'analyse de son texte confirme la prégnance des contextes qui la portent, mais le second degré de lecture informe l'imaginaire social, la culture et les représentations collectives qui ont structuré son contenu. C'est là une caractéristique de la rumeur qui parle un double langage concret et mythique. Derrière l'information qu'elle véhicule s'embusquent des images fixées dans la longue durée des cultures. Dans la rumeur du cachet, ce discours caché vaut à la fois comme descripteur d'une société où les dynamiques politiques étaient envisagées, entre autres, selon les croyances de l'occulte (Bernault \& Tonda 2000:7) et comme métaphore des pratiques souterraines liées à l'activité politique en situation coloniale.

Rumeur du cachet, discours du caché :

de l'invisible et de l'invisibilité en politique

Le caractère heuristique des rumeurs, à la fois comme lieu d'expression des cultures et des imaginaires publics et comme mise en scène orale d'un ordre social ébranlé, n'est plus à établir. Mais le renouvellement de cet usage à propos des rumeurs en Afrique se comprend mieux si on le replace dans le courant des investigations sur la sorcellerie qui ont été relancées depuis une quinzaine d'années, et qui ont promu une perception ouverte de l'imaginaire social africain, accueillant volontiers les logiques du surnaturel et de l'insensé. L'analyse des rumeurs apprend maintenant à écarter la tentation de la vérification des faits et, débarrassée des réticences à l'égard de l'oralité fantastique, elle sonde les univers moins connus des cultures qui leur ont donné naissance (Reumaux 1996; White 2000a). L'étude de la rumeur du cachet peut suivre ce courant épistémologique tant son texte et son édifice imaginaire paraissent s'accrocher au registre des croyances magiques.

11 De manière paradoxale, le cachet de la rumeur qui a d'abord montré une participation au vote en 1960 puis désigné une appartenance politique en 1961 a aussi représenté le caché, l'action souterraine de puissances malfaisantes. À l'évidence, au-delà de la brûlure provoquée par l'acide, quelque chose d'autre était produit par cet objet, dont il fallait impérativement se protéger si l'on en croit les très nombreux symptômes d'inquiétude et réactions de peur qui furent enregistrés dans le pays lors de sa diffusion ${ }^{14}$. Le tampon mettait en danger l'intégrité physique des personnes, mais il menaçait également l'intégrité de leur communauté, politique et familiale. On racontait ainsi qu'une fois apposée sur les épaules de "ceux à qui on donnait de l'argent» (i.e. les «vendus", partisans du PDC ou des partis proches, soutenus par l'administration coloniale), sa 
marque se transmettrait à leur progéniture, c'est-à-dire que «les enfants engendrés naîtraient avec le cachet », estampillés à vie PDC ou Abaprosoma ${ }^{15}$, comme disaient leurs adversaires de l'uprona (Manirakiza, 31 janvier 1990). Ce cachet était, dès l'origine, un objet extraordinaire, étranger, un instrument importé par le Blanc, emblématique de la culture bureaucratique du colonisateur. Mais de surcroît, il opérait une magie hostile lorsqu'il s'appliquait aux corps, et ici l'on est ramené à une autre rumeur lancinante des années 1960-1961, dont le propos était également sous-tendu par les maléfices d'un objet colonial, la seringue. Pendant des mois le bruit courut dans tout le pays que « des enfants étaient morts suite à des piqûres faites au nombril, dans les écoles " par des médecins blancs et " que les Belges vaccinaient les enfants [et les femmes] pour les rendre stériles » (BUR 66/4/1; BUR 74/4: 9 et 10 février 1960). Quelques établissements scolaires se vidèrent et de nombreuses campagnes de vaccination antituberculeuses organisées par les services de la médecine coloniale furent paralysées (BUR 65/1:9 août 1960) ${ }^{16}$. À l'évidence le registre fantasmatique dans lequel s'est épanouie cette rumeur autour des piqûres a aussi servi le développement de la rumeur du cachet ${ }^{17}$. Dans un cas, des représentations négatives de la thérapie belge, qui appartenait pleinement à l'arsenal de la sujétion coloniale, se conjuguaient aux éléments surnaturels associés aux maladies et aux substances susceptibles de les guérir, pour faire de l'injection un empoisonnement fatal (White 2000a : 94-96, 99-102). Dans l'autre, la brûlure de la peau provoquée par l'acide du cachet était également propice à la transmission de tous les poisons, politiques y compris. Dans ce processus filtrait encore l'intervention néfaste des Blancs, qui n'étaient pas directement mis en scène dans la rumeur, mais qui contribuaient à ce nouveau rite de possession des corps et des âmes en stipendiant les partis chargés de cette initiation. À chaque fois, un même imaginaire sorcier traçait les contours d'une coalition de forces occultes occupées à établir une domination physique sur les individus et à asseoir un pouvoir mortifère sur le pays.

De cette «idéo-logique de la sorcellerie » (Bernault \& Tonda 2000: 13; Augé 1975) ${ }^{18}$ affleurant dans la rumeur du cachet, qui dessinait un monde où les rapports de pouvoir se jouaient sur un mode à la fois violent et caché, on peut tirer quelques éléments éclairants sur les perceptions politiques populaires à l'aube de l'indépendance. La matrice de la mort d'abord, et plus précisément de l'extermination (puisque la malédiction du cachet était non seulement mortelle mais encore héréditaire) est courante dans les rumeurs, qui sont la plupart du temps hostiles. Mais elle a enflé dans la rumeur du cachet parce que la cosmologie politique du Burundi monarchique, obsédée par ce que l'on pourrait appeler les abolitions radicales, c'est-à-dire les renversements brutaux de l'ordre politique et social, a été ravivée au moment de la transition à l'indépendance. Cette vision apocalyptique des transitions s'inscrivait dans la longue histoire des interventions funestes des bisuka dévorateurs (les « monstres », les « ogres », les " envahisseurs »), dont les ravages accompagnaient toujours les phases cruciales de l'évolution du royaume depuis les temps précoloniaux, en même temps qu'ils annonçaient sa régénération future (Vansina 1972 : 31-32, 80-82 ; Chrétien 1993c : 394 ; Deslaurier 2002 : 763-770). Au tournant $\mathrm{du} \mathrm{XIX}^{\mathrm{e}}$ et du Xx $\mathrm{x}^{\mathrm{e}}$ siècle, ces êtres puissants et malfaisants avaient fait irruption sous les traits des Blancs, assujettissant le pays et paralysant la monarchie. À l'heure du bouleversement de l'indépendance, quand une ambiance de fin de règne, colonial cette fois-ci, a de nouveau baigné les peurs collectives, on les retrouva dans la propagande des partis nationalistes, UPRONA en tête, toujours occupés à déranger l'ordre des choses dans le pays. Il s'agissait cette fois-ci encore des Blancs, que l'on décrivait en train de faire 
table rase des structures politiques traditionnelles (et, pourquoi pas, de la monarchie, comme ils avaient aidé à le faire au Rwanda), mais ils avaient désormais scellé un pacte malveillant pour perpétuer leur domination sur le pays avec les « vendus » des partis dits démocrates que la propagande uproniste assimilait aussi à des bisuka (Deslaurier 2002 : 763-768). Après les destructions opérées par le système colonial sur l'ordre politique et social du Burundi, l'idée d'un possible anéantissement de la nation par la décolonisation impliquait une résistance acharnée contre les "ennemis" du pays, que ce soit les " envahisseurs " européens ou leurs obligés désignés comme des traitres au mwami (le roi), et l'élimination de tous ces "ogres» ("bisuka») devait finalement assurer le renouveau salutaire du royaume dans l'indépendance. Cette conception magique des bouleversements critiques et la nécessité d'une gestion radicale des tensions politiques imprégnaient les mentalités collectives et ont certainement conditionné pour une part les comportements extrêmes de la population dans le cadre de la rumeur du cachet.

13 Au-delà de la puissante remise en cause de l'économie morale de la colonisation qu'elle traduisait, puisque les Blancs disaient préparer l'émancipation nationale et la liberté politique alors qu'ils détruisaient en réalité les cadres anciens, cette rumeur et ses dimensions surnaturelles renseignent non seulement les représentations populaires de l'invisible, mais encore celles de l'invisibilité qui ont conduit à des phénomènes d'adaptation importants dans le champ des identités politiques. Le paganisme des Burundais dans les années 1960 était religieux, comme s'en plaignaient encore les missionnaires chrétiens, mais il était tout autant politique, au sens où les nouveaux convertis à la démocratie participative de type occidental pratiquaient un polypartisme devant lequel nombre d'observateurs européens restèrent perplexes, mais qui correspondait à une réponse pragmatique face aux rapports de force prodigieux qui se manifestaient alors pour le contrôle du pouvoir. Les sources confirment la multiplicité et la volatilité des affiliations partisanes à l'époque de la décolonisation, avec par exemple des paysans à peine promus au rang de citoyens qui, sommés de se déterminer sur leur appartenance politique, collectionnaient les cartes d'adhésion de partis rivaux pour être toujours en mesure de présenter la plus appropriée à leur situation. Or, dans la rumeur du tampon, le «marquage » identitaire devenait pérenne et décrivait la matérialité - et donc le danger - d'une adhésion politique, voire idéologique, qui n'était pas d'ordinaire assumée ouvertement ou volontairement. Au même titre que dans l'univers sorcier, le diptyque paradoxal du montré et du caché que dévoilait l'apposition d'un cachet sur le corps s'appliquait au monde politique vécu par les Burundais à l'époque, où la libéralisation incomplète de l'activité politique avait favorisé la clandestinisation des pratiques des partis (réunions secrètes), le développement d'une propagande subreptice (tracts, rumeurs), et la dissimulation des opinions et des adhésions politiques du plus grand nombre. Ne nous étonnons pas que cette société politique en respiration souterraine ait été reliée, dans les représentations populaires, au monde de l'occulte. Le nom donné en kirundi aux propagandistes qui œuvraient dans l'ombre faisait ainsi explicitement référence à leur facilité à se mouvoir dans l'obscurité puisqu'on les appelait les bigendajoro, littéralement " ceux qui voyagent la nuit» (Rodegem 1970 : 204). Par la force des choses, leur aisance nocturne témoignait de leur familiarité avec les forces des ténèbres, la nuit étant au Burundi comme ailleurs le domaine par excellence du surnaturel, associée aux images de l'inconnu et des êtres fantastiques, des épreuves et des deuils. Le mélange de crainte et de fascination qui marquait les relations entre les hommes d'un côté, et les esprits du mystère et leurs intermédiaires sorciers de l'autre, 
régissait aussi les comportements prudents et les réactions radicales des premiers vis-àvis des acteurs de la nouvelle vie politique moderne.

La mise en résonance de différentes angoisses traditionnelles et modernes est une constante dans les rumeurs (Morin 1982: 66), et leur impact dépend largement de l'adéquation entre les lointains territoires mentaux qu'elles parcourent et les horizons symboliques qu'elles dévoilent. La rumeur du cachet s'est déployée en tirant substance d'archétypes politiques anciens, plongés dans le chaos d'un imaginaire sorcier qui a activement nourri la compréhension des métamorphoses contemporaines de la scène politique nationale et du désordre provoqué par la compétition entre partis. Cependant elle s'est aussi épanouie parce que son récit a pris sens dans des situations sociales et politiques particulières et qu'il a rencontré, en des lieux et des moments précis, des audiences disposées à l'entendre.

Acteurs, « passeurs » et publicité de la rumeur

15 La prise en compte des articulations entre le développement de la rumeur et son substrat mystique et surnaturel ne suffit pas à expliquer à elle seule son long cheminement et son incroyable essor entre 1960 et 1961. Dans les circonstances les plus brutales de son explosion en particulier, il a fallu, pour que se concrétise la violence structurelle de son message, que des conditions spéciales soient réunies, concernant à la fois les personnes ciblées par le récit, ses "passeurs", et ses audiences publiques. Comme la rumeur compose un mode de communication qui échappe, en partie au moins, au contrôle du pouvoir et compense l'absence de circuits d'information ouverts et transparents, elle forme une instance originale du discours public où l'on peut distinguer la participation des différents corps sociaux au vaste débat de la transformation politique. Aussi, en analysant le rôle de chacun des protagonistes individuels ou collectifs dans les phases cruciales de la rumeur du cachet, qui sont les mieux connues, on peut déterminer les situations sociales et les logiques politiques locales qui ont accru l'intensité et infléchi la forme de ce débat public.

Les boucs émissaires de la rumeur, des figures de la traitrise et de l'ennemi

L'identification des "estampilleurs» présumés est une question centrale pour l'interprétation de la rumeur du cachet. Par un retournement caractéristique en effet, ceux qui étaient présentés comme les agresseurs dans cette rumeur ont été, en réalité, les principales victimes des événements de 1960 et de 1961 surtout, et il semble qu'en éclairant ces cibles véritables, on puisse mieux observer les logiques et les fantasmes qui ont présidé à leur mise à l'index. Il est difficile de connaitre l'identité de toutes les victimes de violences liées à la rumeur, mais dans deux cas significatifs, on dispose de données sociologiques sur les personnes brutalisées, données qui permettent de proposer des hypothèses de lecture sur leur statut et sur ce qu'ils représentaient aux yeux de leurs concitoyens.

17 Sans connaître le détail des accrochages, on sait par exemple que c'est le bourgmestre de la commune de Ngoma, Cyprien Rukuki, qui a été gravement molesté lors des premiers incidents de juin 1961 dans la région orientale de Rutana ${ }^{19}$. Rukuki était un jeune " évolué » en pleine ascension au moment de la transition: encore simple secrétaire indigène au début 1959, il avait été nommé, à 26 ans, sous-chef pendant une année dans la circonscription où il se fit ensuite élire comme bourgmestre en novembre 1960, puis finalement comme député «intérimaire» en janvier $1961{ }^{20}$. Membre et candidat du Rassemblement populaire du Burundi ( $\mathrm{RPB})$, un parti régional d'obédience pédéciste, il 
était à l'échelle locale une figure de proue du Front commun populaire et démocrate, l'alliance électorale menée par le PDC, et il attisa très tôt contre lui l'hostilité de ceux de ses administrés qui soutenaient la cause indépendantiste (BUR 30/1; AE 6.95). Lors des enquêtes menées en 1990 dans la région, qui soulignèrent le fort cloisonnement entre " démocrates » et " indépendantistes », des témoins ont rapporté qu'il « roul[ait] dans un véhicule qu'il n'a[vait] pas acheté ", indiquant à demi-mot qu'il bénéficiait du soutien des Blancs ${ }^{21}$. La charge physique dont il fit l'objet en juin 1961 marqua le repli de sa carrière politique puisqu'il fut abruptement disqualifié par les électeurs lors du scrutin législatif de septembre $1961^{22}$ et qu'il essuya encore de nombreuses attaques dans les mois suivants (AE 6.95).

À Muzinda la victime de la rumeur du cachet s'appelait Térence Kayugi ${ }^{23}$. Kayugi, selon les témoignages, était un cultivateur d'une trentaine d'années travaillant plus au sud dans la commune de Musazi, qui était venu au très populaire marché de Muzinda comme des centaines d'autres paysans le faisaient deux fois par semaine. L'enquête réalisée à Muzinda en novembre dernier a permis d'établir qu'on se souvenait de lui comme d'un militant du PDC (et des partis liés), payé par les Blancs ou par les adversaires de l'UPRONA pour « cacheter » les gens rétifs à l'adhésion au Front commun : selon les témoins, il n'en était pas à sa première tentative lorsqu'il fut tué par la foule. On notera ici que les sources écrites étaient moins définitives à ce sujet, laissant entendre qu'il était peut-être « un uproniste, envoyé pour provocation» (BUR 75/5: [juillet 1961]). Cela rappelle la description des variantes de "l'anti-mythe" dans la rumeur d'Orléans, dont l'une se construit, à l'image de la rumeur elle-même, sur le thème du complot : pour expliquer l'acharnement d'une rumeur sur des pseudo-conspirateurs, on invoque un autre complot encore, fomenté par leurs ennemis dans une volonté délibérée de leur nuire (Morin 1982 : 70-74). Sans se prononcer sur cette hypothèse, on peut néanmoins relever une autre idée qui transparaît dans sa proposition, celle de la duplicité du monde politique, que l'on retrouve dans la description de l'autre victime identifiée de Muzinda, Mathieu Barihaniza, grièvement blessé en tentant d'intercéder en faveur de Kayugi. Les témoins ont en effet expliqué que Barihaniza, «conseiller » de la commune résidant à proximité du cabaret où se déroulait la scène ${ }^{24}$, avait été frappé parce qu'en le voyant intervenir en faveur de Kayugi on l'avait cru retourné et passé au PDC (l'enquête n'a pas permis de clarifier son appartenance partisane). Après tout, n'était-il pas proche du bourgmestre de Muzinda, Boniface Ntamirukiro, qui s'affichait uproniste mais rencontrait en secret des individus, comme Kayugi justement, chargés de "recruter» avec leur cachet des membres pour les formations pédécistes ${ }^{25}$ ?

Bien qu'ils ne soient pas similaires, les profils des victimes des lynchages populaires de 1961 ont des traits communs qui caractérisent bien les fantasmes de la société burundaise de l'époque, bouleversée par les changements à l'œuvre dans le cadre de la transition politique, mais aussi par le renversement des rapports de force qui s'est dessiné en faveur de l'upRona au milieu de cette année-là. Rukuki à Rutana et Kayugi dans la province de Bubanza ne fréquentaient peut-être pas les mêmes cercles sociaux puisque l'un émargeait au tableau des responsabilités administratives quand l'autre travaillait aux champs, mais ils incarnaient chacun à leur manière le "traitre" et le "vendu", deux figures essentielles de la rhétorique politique burundaise qui les ont pratiquement condamnés. mener la propagande du PDC ou du Front commun, des gens capables «de trahir leur père, leur mère et leurs enfants au prix d'argent, pourvu que leur estomac soit satisfait et 
leurs poches remplies " (BUR 65/1: [27 août 1960]). La propagande de l'UPRONA depuis 1960 avait ressassé ce thème, en repopularisant même à l'encontre de ses adversaires le surnom de Bansumirinda (littéralement « ceux qui cherchent de quoi approvisionner leur ventre»), et elle avait instillé une comparaison péjorative entre ces derniers et les Rwandais qui avaient « vendu leur pays » et "versé le sang de leurs frères [en] préférant leur ventre" au moment de la "révolution sociale» de novembre 1959, activement soutenue par les autorités coloniales belges (BUR 65/1: 12 octobre 1960; Deslaurier 2002: 768-769). À la corruption par les Blancs des pédécistes et d'autres Abaprosoma, comme ont pu être appelés Rukuki et Kayugi, se conjuguait par ailleurs une trahison suprême, celle de l'insoumission au mwami (le roi). Par un jeu subtil de cascades identificatoires, l' UPRONA, dont le leader n'était autre que le fils du mwami, Louis Rwagasore, était parvenue à se présenter comme le parti des «fidèles " tandis que ses concurrents étaient qualifiés de bamenja (les rebelles), une catégorie politiquement connotée, à l'ancrage historique lointain, et qui comportait au début des années 1960 une valeur dépréciative ${ }^{26}$. Comparés à Kilima, illustre renégat qui avait nargué le mwami Gisabo (l'arrière grand-père de Rwagasore) et contesté sa légitimité face aux Allemands à la fin du XIX ${ }^{\mathrm{e}}$ siècle, ce sont surtout les leaders du PDC, pour la plupart issus d'une branche dynastique parallèle à celle de Rwagasore, celle des Batare, qui ont subi cette campagne de dénigrement. Leur position face à la colonisation et des incidences généalogiques, territoriales et politiques, rendaient opérante une qualification sous-tendant leur dissidence (Deslaurier 2002: 755-762). Avec eux, l'ensemble des membres des partis du Front commun étaient assimilés à des révoltés et, en définitive, tous ceux qui n'étaient pas des badasigana (nom donné aux upronistes, se référant aux combattants du mwami Gisabo) ne pouvaient être que des « ennemis » (" abansi ») du mwami et du Burundi, des traitres à la monarchie qui vendaient leur pays aux Blancs comme l'avait fait en son temps Kilima (ibid.). L'insulte était grave, surtout couplée à une allusion au Rwanda voisin où la "révolution sociale » hutue avait abouti à l'établissement d'un régime républicain (janvier 1961). Contrairement au cas rwandais, le mwami était encore très populaire dans les milieux paysans comme dans les milieux "évolués» du Burundi de la décolonisation et les ressorts de la tradition et de la fidélité monarchiques ont été actionnés par tous les partis burundais pour recueillir l'adhésion populaire - l'UPRONA ayant sur ce point une bien plus grande efficacité que le PDC.

21 Que criait la foule lorsqu'elle s'est emparée de Kayugi et l'a conduit au bûcher? «À mort les traîtres, les républicains, ceux qui ont vendu le pays, ceux qui trahissent le Mwami » ( BUR 74/4 : 28 juin 1961). Quelle explication vaguement fataliste ont donné les témoins du drame à la radicalité du traitement réservé au jeune cultivateur ? C'était un « ennemi » ( umwansi ») du mwami, un « rebelle» («umumenja»), qui a été «mal tué» mais dont le sort était joué à partir du moment où ses activités de lèse-majesté avaient été découvertes ${ }^{27}$. Quel argument a été utilisé contre le premier édile de Ngoma, Rukuki, par ses administrés, pour demander sa mise à l'écart? «Parce qu'il n'accepte pas le mwami, nous le refusons comme bourgmestre» (AE 6.95: 10 novembre 1961). Par-delà leur opposition commune à l'uPRoNA, les victimes de la rumeur du cachet représentaient surtout une menace pour la monarchie, perçue à l'époque comme la seule capable de maintenir l'équilibre d'un Burundi redevenu indépendant en lui épargnant les crises sanglantes de son voisin rwandais. En filigrane émergent les tensions entre la tradition politique burundaise et la modernité du nouveau jeu multipartite que la rumeur du cachet parvenait à associer en mettant au diapason du mythe du complot contre le mwami 
l'actualité de la lutte partisane. Kayugi et Rukuki incarnaient hic et nunc le fantasme populaire du renversement de la monarchie et le péril de ces moments où des monstres coalisés (les bisuka, mais aussi les bamenja) ébranlaient le pays. Ils ont chacun rempli «la case vide du coupable à double visage » dans la crise politique du moment (Morin 1982 : 50-51) parce qu'un héritage historique accompagnait le regard que les gens portaient sur leur conduite publique et que des éléments concrets, parfois arrachés à leur contexte, ont en quelque sorte validé leur culpabilité. La rumeur disait qu'ils se servaient d'un cachet magique pour éliminer leurs ennemis ou faire entrer les gens dans un nouvel ordre politique sur lequel les Blancs établissaient leur puissance par l'argent. Or l'évolué Rukuki travaillait depuis plusieurs années au contact des Européens comme auxiliaire dans l'administration coloniale : il s'était enrichi grâce à cette proximité et représentait l'ordre politique moderne qui refusait le pouvoir du mwami et les autorités traditionnelles ( $\mathrm{AE}$ 6.95 : lettres de 1961). De son côté, Kayugi ne frayait pas avec les Blancs, mais ses activités concourraient à leur domination et par ailleurs son statut personnel le situait en marge de l'environnement social de Muzinda, ce qui a sûrement aggravé les soupçons quant à son comportement. En particulier il n'était pas de cette commune mais de celle voisine de Musazi, où d'ailleurs il n'était pas vraiment « installé » puisqu'il travaillait sur les terres d'un tiers et habitait avec sa jeune épouse chez une autre personne ${ }^{28}$. Surtout, il était étranger à la région où il s'était établi une dizaine d'années auparavant. Avant de rejoindre la plaine occidentale du Burundi il était originaire du versant nord-oriental du pays, dans la région de Kirundo. Or, si l'on se reporte aux configurations mentales qui pouvaient être celles de 1961 vis-à-vis de cette région, il s'agissait d'une zone troublée et politiquement active, où Ntidendereza, l'un des grands leaders du PDC, exerçait son autorité de chef avant de devenir ministre de l'Intérieur en janvier 1961. C'était une région difficile pour les upronistes, sinon dissidente puisque la branche familiale des Batare dont Ntidendereza était un membre éminent y maintenait une farouche opposition au "parti loyal », c'est-à-dire à l'UPRONA (Deslaurier 2002: 1081-1082). En dehors de ses options politiques, le jeune Kayugi cumulait donc sur sa personne les désavantages attachés non seulement à sa position d'étranger dans la communauté locale (un «Autre » déstabilisant et menaçant, mais aussi plus vulnérable), mais encore à la proximité qu'on pouvait lui prêter avec les Batare du PDC. On notera ici que les considérations identitaires à l'œuvre pour faire de Kayugi comme de Rukuki des boucs émissaires parfaits ont été sociales, politiques, territoriales et éventuellement claniques. Mais elles n'ont pas recouvert de critères ethniques en tant que tels : Kayugi en effet était un paysan hutu tandis que Rukuki était un évolué tutsi, et ni la documentation, ni les témoignages ne prêtent de rôle aux identités ethniques dans cette affaire. C'est avant tout dans la dialectique de la fidélité ou de la traîtrise vis-à-vis du mwami que se sont inscrits leur isolement politique et leur brutalisation, une dialectique qui recoupait aussi celle du respect dû aux autorités « coutumières » et de la résistance au pouvoir colonial et à ses aménagements politiques modernes.

Dans la compréhension de cette soudure administrative et politique que fut la décolonisation burundaise, la rumeur renseigne la puissance de la foi monarchique qui a formé, dans un contexte mouvementé et incertain, un garde-fou contre les aléas de la modernisation. La désignation d'individus et, par devers eux, de groupes se détachant de la société ordinaire par leurs relations avec les Blancs et leur insoumission présumée à l'autorité monarchique, a certainement permis de résoudre un besoin d'unanimité, revigoré par un sentiment général d'insécurité en période d'incertitude politique (Morin 
1982 : 9). On doit toutefois s'interroger sur ces anxiétés et ces réactions collectives qui suggèrent une vision du monde unifiée et homogène, et il faut alors questionner la rumeur sous l'angle des modalités de sa diffusion et des réseaux du discours public.

Rumeur et propagande politique : les réseaux de circulation

Étroitement liées à la question des personnes visées par la rumeur, les interrogations sur ses « passeurs » et ses « milieux conducteurs » ouvrent des perspectives intéressantes sur le plan des théories de la communication politique et sur celui des interactions entre rumeur et propagande. Aux représentations courantes des rumeurs est attachée l'idée d'une communication de masse qui se marie aux notions d'anonymat, d'anarchie et de crédulité (Froissart 2001 : 49) et au Burundi en particulier, on les imagine souvent circuler dans une paysannerie compacte, inculte et analphabète. Les études sociologiques pourtant, depuis Morin (1982) jusqu'à Reumaux (1996), ont montré que les publics des rumeurs étaient nettement différenciés, que des «influents» ou des "surinformés » jouaient un rôle manifeste dans la structuration des audiences, et qu'une exposition sélective à des thèmes prédéfinis préparait aussi très bien l'organisation du débat public (ibid.). Ce qui ressort du cas burundais ressemble, en fait, à un tableau nuancé des acteurs, des voies et des flux de la communication.

Les éléments collectés à propos des diffuseurs de la rumeur du cachet laissent penser que des militants du parti UPRONA ont directement agi sur sa diffusion, voire l'ont fait naitre. Ainsi, dans sa phase initiale à Ngozi, à la fin 1960, des officiers de police coloniaux ont arrêté «le nommé Ndikumagenge Salvator, propagandiste UPRONA, prévenu d'être à l'origine de cette propagande [du cachet] » (BUR 61/2:28 novembre 1960) et comme on va le voir, ce dernier a sans doute bien joué un rôle lors d'une phase cruciale du développement de la rumeur. On se souvient d'abord que les abstentions dans les communes où la rumeur du cachet est apparue pour la première fois semblaient liées à un mot d'ordre uproniste local, massivement suivi par les électeurs, sinon par conviction idéologique du moins par persuasion fantastique. Ensuite, en matière de persuasion, Ndikumagenge disposait d'atouts qui faisaient de lui le rhéteur sûrement le mieux qualifié et le plus écouté de cette région. Jouissant du prestige et de l'autorité que lui conférait son statut d'évolué, il était en outre le secrétaire personnel de Louis Rwagasore lorsque ce dernier prit en 1959 la tête de la chefferie du Butanyerera, où se situaient précisément les communes réfractaires au vote (BUR 65/1: 6-12 juin 1960). Proche assistant du prince, Ndikumagenge était d'une certaine manière aussi son porte-parole local et celui de son parti, l'uprona, et quand le leader charismatique était absent de la région, les habitants le considéraient comme son représentant. Comme à l'époque des élections communales les autorités coloniales avaient neutralisé Rwagasore en l'assignant à résidence au sud du pays, les consignes diffusées par Ndikumagenge ont vraisemblablement été considérées comme provenant du prince confiné.

En suggérant cette lecture, on conforte la théorie d'une audience captive de «l'influent " qui éclaire sur un mode interactionniste l'une des facettes sociologiques de l'économie des rumeurs. En même temps, on conjecture aussi une manipulation volontaire de ce genre conversationnel particulier, à des fins de propagande politique ou comme instrument de la lutte entre les élites politiques, puisque la rumeur détermine les positions d'exclusion ou de leadership au sein des groupes où elle circule (Gluckman 1963). Mais si des indicateurs vont effectivement dans ce sens pour la rumeur du cachet, on ne résout pas pour autant toute l'équation de sa diffusion et de son explosion dans le pays. L'idée que des leaders aient pu élaborer des stratégies de communication politique 
fondées sur l'information menaçante de la rumeur ne peut être écartée, mais il est impossible qu'ils aient pu maîtriser de bout en bout un phénomène aussi volatile. De nombreux bruits ayant pour toile de fond le rejet d'une initiative coloniale ont été attribués par les fonctionnaires belges aux propagandistes nationalistes et, de fait, la nature subversive des rumeurs concordait bien avec le programme d'opposition des partis indépendantistes. Certaines ont d'ailleurs ressemblé à de véritables opérations de déstabilisation de l'État colonial, menées avec très peu de moyens (Scott 1985) : à côté de la rumeur du cachet et de ses conséquences électorales, celle des piqûres avec le refus des campagnes de vaccination ou encore les bruits sur l'exonération fiscale à laquelle pouvaient prétendre les upronistes ${ }^{29}$, sont de ces exemples où il serait angélique de minimiser l'implication des militants nationalistes. Bien évidemment, ces derniers ont profité de positions privilégiées dans l'administration coloniale et coutumière, et ils ont utilisé les réseaux familiers de transmission de l'information sur les collines (où l'habitat dispersé a toujours encouragé les échanges de proximité) pour mener ce que les fonctionnaires belges appelaient des «campagnes de faux bruits » (BUR 65/1: 8 juillet 1960) - façon d'exprimer ces interférences si fréquentes entre rumeur et propagande ${ }^{30}$. Cependant, on pense que l'action des propagandistes s'est située à la bascule entre le mot d'ordre et la rumeur, et que leur responsabilité était restreinte au colportage des « faux bruits ", sans qu'ils en soient forcément à l'origine. Quand bien même on pourrait les raccrocher aux premiers maillons de la chaîne de gestation et de propagation des rumeurs, leur rôle se limitait en fait à l'adaptation et à la transformation d'un contenu déjà à l'esprit des auditeurs. Aussi, si la multiplication des intermédiaires qualifiés peut donner l'impression que l'exploitation des rumeurs était organisée, en réalité elle n'informe que la densité marquante d'influents dont disposait l' UPRONA (ou, moins souvent, ses alliés) au sein de milieux prédisposés à entendre ses discours, et démontre seulement que ses propagandistes maniaient avec brio des outils de sédition forgés dans des certitudes collectives (Deslaurier 2002: 755). Très prosaïquement par ailleurs, on observera qu'en imputant aux activistes upronistes la plupart des rumeurs contestataires en circulation dans le pays, les autorités coloniales évitaient non seulement d'y lire leur propre mise en accusation, mais encore trouvaient prétexte à écarter les plus dynamiques de leurs adversaires.

Après l'arrestation de Ndikumagenge, la rumeur du cachet a poursuivi son expansion et de proche en proche des militants-relais ont répercuté son message, récupéré ses avantages politiques et parfois aussi subi ses conséquences judiciaires. Toutefois, de Ngozi à Bururi et de Rutana à Bubanza ces «passeurs » ont accompagné le mouvement de la rumeur plus qu'ils ne l'ont canalisé, et le constat selon lequel elle n'a pas pris partout la même ampleur soulève la question de la fertilité des terreaux qui l'ont vu germer. Il est sûr qu'une culture politique commune où l'aversion pour les « vendus » n'avait d'égal que le ressentiment vis-à-vis du système colonial et la crainte des magies malfaisantes a d'emblée fourni le socle de son expansion à l'échelle nationale. En dehors de ses principaux points d'impact, dûment renseignés par les documents écrits, on dispose en effet, pour suivre son avancée, de témoignages oraux qui offrent des localisations très variées et donnent l'image de sa généralisation en quelques mois. Cette généralisation, dans des milieux sociologiques forts différents, convainc de la participation plurale des groupes sociaux à la structuration du débat public et politique à travers la rumeur. $\mathrm{Ce}$ mode de communication fondé sur la sociabilité et sur les échanges de proximité a traversé tous les corps sociaux en donnant une voix à ceux qui d'ordinaire n'en avaient 
pas (Scott 1985 : 282-290), et dans le Burundi rural il a été encouragé par des réseaux de voisinage et des dispositifs de transmission orale rôdés depuis des siècles par les contraintes de l'habitat dispersé sur les collines (Deslaurier 2002: 668). Mais il reste à expliquer pourquoi la rumeur, banalisée sur le territoire national, n'a pas provoqué partout les mêmes phénomènes de contestation ou de violence. Une réponse est que le processus cumulatif de sa circulation a aggravé ses conséquences dans le temps: les incidents initiaux liés au cachet, en concrétisant la réalité d'un danger quelque part, ont pu générer ailleurs des répliques plus vives. Néanmoins cet argument est partiel puisqu'il s'échafaude autour du rythme chronologique de la propagation de la rumeur sans cerner les conditions de son impérialisme sur quelques foyers territoriaux. Pourquoi, par exemple, les taux d'abstention lors des élections communales ont-ils été plus élevés dans le territoire de Ngozi que dans les régions voisines, alors que la rumeur du cachet y était également répandue et que le vote eut lieu à quelques jours d'intervalle seulement? Pourquoi l'escalade de la rumeur a-t-elle tué à Muzinda, alors qu'une semaine auparavant elle a uniquement blessé à Makamba et Rutana ? Inévitablement les dimensions spatiales du phénomène s'imposent pour expliquer ces différences : l'histoire et les dynamiques locales ont créé des environnements plus ou moins propices à la déflagration de la rumeur.

Les occurrences locales de la rumeur

On peut admettre avec Edgar Morin (1982: 7) qu'il convient en général d'interpréter «le phénomène de la rumeur non pas dans le cadre localisé, mais dans le cadre socialisé » des espaces où elle s'énonce, en considérant avec lui que les événements liés à la rumeur sont révélateurs d'une structure psychologique générale. Cet article s'est d'ailleurs employé à souligner l'importance du substrat socio-historique et culturel qui a irrigué l'imaginaire et les mentalités politiques burundaises dans l'élaboration des figures évolutives de la rumeur. Cependant, on ne peut pas faire abstraction des variables spatiales de l'explosion des rumeurs qui laissent supposer qu'en certains lieux des mécanismes stimulateurs ont été plus puissants qu'ailleurs ou, en inversant le propos, que des processus « inhibiteurs » y ont sûrement été moins efficaces.

La description des situations politiques dans les quatre zones où des épisodes brutaux en lien avec la rumeur du cachet ont été observés (Ngozi, Makamba, Rutana et Bubanza) ne permet pas d'envisager une région «type » où les risques de déflagration auraient été dominants. Néanmoins, ces cas de figure mettent en lumière une caractéristique commune : la fragilité. Tous ces sites appartenaient en effet à des périmètres de haute tension entre obédiences partisanes ou, de manière plus paradoxale, ils formaient des zones d'unanimité politique au cœur de régions hostiles. Ainsi, les zones des échauffourées de mai-juin 1961, autour de Makamba et de Rutana, appartiennent à des espaces géographiques plus larges où la compétition politique a été animée et où les oppositions entre "démocrates" et "nationalistes", recouvrant des rivalités personnelles ou confessionnelles, se sont parfois manifestées dans de vifs accrochages physiques (Deslaurier 2002 : 927-950). En revanche, le carré géographique composé des communes de Mubogora, Gaheta, Rango et Butaganzwa, où la rumeur est apparue pour la première fois dans une forme publique et opérante, ainsi que la commune de Muzinda, où s'est déroulé l'événement le plus grave, constituaient chacun des sanctuaires politiques monocolores à l'intérieur de régions disputées. Dans le territoire de Ngozi où les frictions politiques n'ont pas manqué, les quatre communes où la rumeur provoqua l'abstention en 1960 dressaient un véritable rempart uproniste face au front des communes pédécistes 
voisines. Dans ce territoire en effet les chefs Ntakiyica et Baranyanka surtout, princes de la lignée des Batare proches du pouvoir colonial ${ }^{31}$, avaient établi une influence dominante du PDC qui s'était trouvée remise en cause à partir du moment où le prince Rwagasore, fils du mwami et figure emblématique de l'UPRONA, était devenu chef à leurs côtés, dans le Butanyerera. Là, en quelques mois, les administrés furent acquis à l'UPRONA, et ils rivalisèrent avec leurs voisins pédécistes. À Muzinda, la configuration politique générale était assez similaire. Le territoire de Bubanza qui abritait cette commune était traversé par des influences partisanes diverses, avec des tensions marquées entre certains partis « populaires ", rattachés à la sphère des démocrates, et les formations nationalistes (l'upRona, mais aussi l'UnARU, Union nationale africaine du Ruanda-Urundi). Mais, Muzinda et les communes environnantes formaient dans ce territoire un bastion uproniste intérieur qui s'était largement construit sur l'opposition au chef local Nyarusage, du Front commun, encouragée par son voisin émargeant à l'UPRONA, le chef Bankamwabo.

Les deux sites où la rumeur du cachet a eu les répercussions les plus spectaculaires étaient donc caractérisés par des situations de domination politique que la manipulation des étiquettes partisanes au moment des élections n'a dissimulées que temporairement ${ }^{32}$. Il n'est pas innocent que la rumeur ait incubé et plus tard explosé précisément dans ces fiefs upronistes : ce sont les fragilités de cette situation dominante qu'elle soulignait et ses déséquilibres qu'elle faisait craindre. À ce sujet, l'explication fournie par le ministre pédéciste Ntidendereza à propos de «la fable du cachet » à Muzinda est éclairante : selon lui, Kayugi était un propagandiste de l'UPRONA dont la provocation aurait eu "pour but d'éprouver la fidélité d'une population entièrement uproniste, mais pour qui l'UPRONA aurait craint l'influence de deux personnalités de la commune ", affiliées à des partis populaires ou démocrates (BUR 74/4: 28 juin 1961). "Un fanatisme farouche [et] une volonté obstinée d'abattre tout ce qui n'est pas uproniste " auraient ainsi trouvé leur tragique expression dans l'incident du 25 juin à Muzinda, et l'uprona aurait un peu partout « utilisé des données locales » de cette manière, « aux fins de créer des incidents [répondant] à un plan d'ensemble couvrant tout le pays » (ibid.). L'idée d'un plan global d'intimidation rejoint celle du complot déjà mentionnée, difficile à certifier surtout quand tous les témoignages concordent pour dire que Kayugi était démocrate et non pas uproniste. Mais de facto on peut noter que partout où la rumeur est passée en force, c'est l' UPRONA qui a profité de ses effets sur le plan de l'identification politique, c'est-à-dire que la rumeur a bénéficié au parti qui était déjà le plus puissant dans les sites où elle a explosé. Elle a servi dans ces zones à conforter un unanimisme politique menacé par les incertitudes des affiliations alentour et consacré par l'élimination des quelques figures locales qui pouvaient lui faire opposition. Dans ces conditions l'arrestation des militants upronistes soupçonnés d'être impliqués dans les « mises en scène » du cachet, plutôt que de faire fléchir la fidélité à l'uPRONA, a redoublé la conviction collective d'être dans le «bon" parti : une forme de martyrologie politique s'est constituée dans ces régions autour de la glorification des militants ayant fait les frais de la coercition du pouvoir blanc et plus globalement des populations ayant résisté par tous les moyens, y compris violents, à l'emprise coloniale et à la poussée des partis souhaitant son maintien ${ }^{33}$.

La rumeur du cachet a fait son chemin partout au Burundi parce qu'un imaginaire social, des archétypes politiques et une mémoire collective étaient partagés par sa population entière. La prégnance de cette unité historique et politique, souvent citée pour exemple de l'intégration nationale à l'époque des décolonisations africaines, n'a toutefois pas régi 
partout les mêmes comportements politiques et les mêmes mobilisations morales ou idéologiques. Le caractère déterminant des dynamiques locales de suprématie politique introduit le paradoxe d'une rumeur qui d'un côté peut être considérée comme une arme du faible, un instrument de combat politique pour les groupes écartés du débat public (Scott 1985 : 282-290), mais de l'autre ne sort ses effets les plus lourds que maniée dans un contexte où ces groupes dominent la situation. Dire que la rumeur du cachet était seulement un discours de subversion contre le système colonial et une parole commune de résistance serait donc incomplet: elle était aussi une inquisition collective, une instance de vérification de l'orthodoxie des appartenances politiques.

Comme les rumeurs forment un genre conversationnel à part entraînant l'ensemble de la population dans de formidables opérations de sociabilité, où la création et le maintien de liens sociaux sont primordiaux, elles composent un régime d'information qui ne promeut pas toujours les mêmes valeurs que le discours produit par les informations officielles ou les médias. Aussi l'étude d'une rumeur autant généralisée que celle du cachet, liée au processus de l'émancipation burundaise, permet-elle d'entrevoir les représentations communes du pouvoir au moment de la décolonisation et de comprendre comment les gens regardaient l'État colonial à l'approche de sa disparition. Les apports de cette analyse à la compréhension du politique au Burundi vont au-delà du simple constat de l'efficacité de la rumeur du cachet dans certaines circonstances, en soulignant la structuration historique des éléments politiques, sociaux et culturels qui ont rendu possible sa circulation. Grâce aux traces proprement historicisées de la rumeur, qui sont présentes dans les bulletins de la Sûreté et dans les témoignages oraux avec des datations précises, on a notamment accès aux imaginaires des populations qui se situent sur des terrains d'ordinaire difficiles à explorer de façon rétrospective. Avec sa part d'incroyable, on découvre un monde perçu où des forces surnaturelles canalisaient les possibles politiques du pays, et dans son apparente fantaisie, on lit le recodage local de la réorganisation des hiérarchies du pouvoir dans le nouvel ordre promu par les colonisateurs en partance (White 2000a: 210-211). Enfin l'implication des différents groupes sociaux dans cette conversation générale témoigne de leur rôle dans la structuration d'un débat public pointant le changement social et accusant la désintégration d'une unité nationale fondée sur la fidélité monarchique: la parole politique de la rumeur n'était réservée ni au combat des «faibles » ni à la compétition entre élites; elle était le bien public peut-être le plus démocratiquement partagé de l'époque. 
1975 Théories des pouvoirs et idéologie : étude de cas en Côte-d'Ivoire, Paris, Hermann.

BAgANZiCAHA, M. \& LE JeUne, G.

1979 « La presse au Rwanda et au Burundi à la fin de la Tutelle », Culture et société, 2 : 11-31.

BERNAULT, F. \& TONDA, J.

2000 « Dynamiques de l'invisible en Afrique », Politique Africaine, 79 : 5-16.

BUKURU, P.

1990 L'organisation et le déroulement des élections communales et législatives au Burundi (1960-1961), Mémoire de l'Université du Burundi, Bujumbura, Faculté des Lettres et Sciences humaines.

CHRÉTIEN, J.-P.

1993a Burundi, l'histoire retrouvée. 25 ans de métier d'historien en Afrique, Paris, Karthala.

$1993 b$ « Une révolte en 1934. Les racines traditionalistes de l'hostilité à la colonisation », in J.-P. CHRÉTIEN, op. cit. : 219-274.

1993c « Les traditionnistes lettrés du Burundi à l'école des bibliothèques missionnaires (1940-1960)», in J.-P. CHRÉTIEN, op. cit. : 379-402.

Deslaurier, $\mathrm{C}$.

1998 « Du nouveau pour l'histoire politique du Burundi à la veille de l'indépendance : la documentation secrète de la Sûreté (1958-1961) », Cahiers du CRA, Paris, CRA, Université de Paris $1: 9: 39-69$.

2002 Un monde politique en mutation : le Burundi à la veille de l'indépendance, 1956-1961, Thèse de doctorat, Université de Paris 1 (à paraître en 2005, Paris, Karthala).

FROISSART, P.

2000 "L'invention du "plus vieux média du monde" ", MEI Médiation et information, 12-13 : 183-195.

2001 "Penser les médias sans notion de masse », Actes du $12^{e}$ Congrès national des sciences de l'information et de la communication, Émergences et continuité dans les recherches en information et communication, Paris, UNESCO : 49-56.

GLUCKMAN, M.

1963 « Gossip and Scandal », Current Anthropology, 4 (3) : 307-316.

HARROY, J.-P.

1987 Burundi 1955-1962, Souvenirs d'un combattant d'une guerre perdue, Bruxelles, Hayez.

MORIN, E. et al.

1982 [1969] La rumeur d'Orléans, Paris, Éditions du Seuil (« Points-Série Essais »).

ReUMAUX, F.

1996 La veuve noire. Message et transmission de la rumeur, Paris, Méridiens Klincksieck.

RODEGEM, F. M.

1970 Dictionnaire rundi-français, Tervuren, Annales du MRAC.

ScOTT, J. C. 
1985 Weapons of the Weak : Everyday Forms of Peasant Resistance, New Haven-London, Yale University Press.

VANSINA, J.

1972 La légende du passé. Traditions orales du Burundi, Tervuren, MRAC, Archives d'anthropologie (16).

WHITE, L.

2000a Speaking with Vampires. Rumor and History in Colonial Africa, Berkeley, University of California Press.

$2000 \mathrm{~b}$ « Histoire africaine, histoire orale et vampires. Procès et palabres à Kampala dans les années $50 »$, Politique Africaine, $79: 83-100$.

sources

Archives africaines de Bruxelles (AAB)

AI $4369 / 4$

11 janv. 1961 Knops, Halleux et Roggen, « Rapport de la commission d'observateurs pour les élections communales du Burundi (novembre-décembre 1960) », Bruxelles.

BUR $30 / 1$

Dossier administratif de Cyprien Rukuki.

BUR $60 / 2$

6 févr. 1960 Rudipresse, Usumbura, ${ }^{\circ} 155$.

20 févr. 1960 Amakuru y'Uburundi, Kitega, $\mathrm{n}^{\circ} 1$.

$\mathbf{1}^{\mathrm{er}}$ mars 1960 Amakuru y'Uburundi, Kitega, $\mathrm{n}^{\circ} 2$.

$\mathbf{1}^{\mathrm{er}}$ juill. 1961 «Incident à Muzinda en territoire de Bubanza », Rudipresse, Kitega, $\mathrm{n}^{\circ} 230: 4$.

BUR $61 / 2$

28 nov. 1960 Bulletin d'information (BI) 255 de la Sûreté, Kitega.

BUR $65 / 1$

26 févr. 1960 BI 87 de la Sûreté, Usumbura.

24 mai 1960 BI 107 de la Sûreté, Kitega.

6-12 juin 1960 Rapport hebdomadaire (RH) de la Sûreté, Usumbura.

8 juillet 1960 BI 145 de la Sûreté, Kitega.

9 août 1960 BI 185 de la Sûreté, Kitega.

[27 août 1960] Tract de l'UPRonA, Barundi, Barundikazi mwese, Badasigana mu majambere bo hirya no hino... (Burundais, Burundaises, upronistes de tous côtés...), s.l. 
12 oct. 1960 Tract de l'upronA, Badasigana b'Imana n'Uburundi... (Upronistes de Dieu et du Burundi...), Usumbura.

BUR $66 / 4 / 1$

9 févr. 1960 Note manuscrite adressée au Représentant de l'autorité tutélaire (RAT) à Usumbura par l'agent G. Culot, Usumbura.

BUR 74/4

10 févr. 1960 BI non numéroté de la Sûreté, s.l. [Usumbura].

2 mai 1960 BI 72 de la Sûreté, Kitega.

2 juin 1960 BI 116 de la Sûreté, Kitega.

28 juin 1961 «Rapport sur les incidents du 25 juin en commune de Muzinda » rédigé par le ministre de l'Intérieur J.-B. Ntidendereza, Kitega.

BUR $75 / 5$

10 avr. 1961 « Feuille d'audience et de jugement » contre M. Tuyaga et V. Muderenganyo (propagandistes de l'uprona) signée par le juge de police A. Cappuyns, Ngozi (avec le témoignage du bourgmestre pédéciste de la commune Gaheta sur les problèmes causés par la rumeur du cachet au moment du scrutin communal de 1960).

[juillet 1961] Note manuscrite anonyme comprenant des renseignements fournis par l'AT de Bubanza, s.l. [Usumbura], s.d. [juillet 1961].

BUR $243 / 1$

13 mars 1961 « Rapport sur l'information en Urundi » par l'Agent territorial principal J. Mangelinckx, Usumbura.

Archives nationales du Burundi (ANB)

AE 6.92 et 13.32 , AG 10.2 , BG1 6.22, 6.31, 6.42, 6.62, et 8.5

1958-1965 Documentation diverse concernant Cyprien Rukuki, bourgmestre de Ngoma (Rutana).

AE 6.95

10 nov. 1961 Lettre adressée au mwami, au $1^{\mathrm{er}}$ ministre, à l'administrateur de province et aux députés de Rutana par « les notables, la jeunesse, les simples gens » (10 signataires), à propos du bourgmestre Cyprien Rukuki.

\section{Entretiens}


31 janv. 1990 Manirakiza, interrogé en kirundi par Pamphile Bukuru (1990 : 89), Gihinga.

6 févr. 1990 Sakubu, interrogé en kirundi par P. Bukuru (1990 : 88), Kibumbu (Muramvya).

11 oct. 1990 Séverin Ruduri, interrogé en français par mes soins, Gitanga (Rutana).

11 nov. 1990 Enquête collective menée en kirundi auprès d'anciens catéchistes de la mission de Mpinga (Rutana) avec C. Sindahabaye et A. Nabucencwa du Centre de civilisation burundaise (Bujumbura), Mpinga-Kayove (Rutana).

$1^{\text {er }}$ nov. 2004 Abdallah Juma, Ruhokeye et Mukuvyi, interrogés en kirundi avec l'aide et la traduction de C. Ayubu et H. Mbonimpa, Muzinda (Bujumbura-Rural).

\section{NOTES}

1. Après une courte occupation allemande, les deux vieilles monarchies du Burundi et du Rwanda ont été placées sous mandat de la Société des Nations au lendemain de la Première Guerre mondiale, puis sous tutelle des Nations-Unies après la Seconde Guerre mondiale. Administrés conjointement pendant plus d'un demi-siècle par la Belgique, ces deux pays qui formaient le « Territoire du Ruanda-Urundi » accédèrent séparément à l'indépendance le $1^{\mathrm{er}}$ juillet 1962 . Le Rwanda fit son entrée dans le concert des nations indépendantes avec un régime républicain installé en 1961 avec l'appui des colonisateurs, tandis que le Burundi maintint sa royauté, constitutionnalisée à la manière belge.

2. Entre la fin 1959 et la fin 1961, 26 partis politiques ont été agréés par l'administration coloniale.

3. La thèse de doctorat que j'ai soutenue en 2002 comporte de plus amples développements sur la propagande politique, ses voies de diffusion et sa réception par le public burundais (DESLAURIER 2002).

4. Le terme ibihuha désigne en kirundi les rumeurs mensongères, mais il possède un synonyme, urukurukuru, qui inscrit plus volontiers ce genre d'information dans l'ordre de la véracité. La construction du mot l'apparente en effet aux « nouvelles » (amakuru), qui s'opposent formellement aux récits de fiction et sont souvent porteuses de l'information officielle (RODEGEM 1970 : 175, 250). On dit d'ailleurs que « la rumeur c'est la nouvelle » (Urukurukuru niyo nkuru).

5. Même si les supports de l'information se sont diversifiés à l'heure de la décolonisation, on n'a pas assisté à l'explosion d'une vie médiatique moderne et autonome, au sens où des mass media auraient été les vecteurs essentiels des messages politiques. L'importance de la radio était limitée à l'époque (HARROY 1987 : 529 ; BUR 243/1 : 13 mars 1961), tandis que l'offre de presse écrite est demeurée restreinte, dominée par les institutions religieuses et le gouvernement colonial qui contrôlaient la plupart des outils de l'impression périodique (BAGANZICAHA \& LE JEUNE 1979 : 14-15).

6. Les informations relatives à la rumeur du cachet ont été fournies de manière spontanée par les personnes interrogées, alors que la discussion portait sur la propagande politique en général. Cette orientation de la conversation souligne l'importance qu'a eue la rumeur à l'époque. Elle est d'ailleurs célèbre par-delà les générations qui ont vécu la décolonisation, comme me l'ont prouvé de nombreuses conversations au Burundi. 7. Toute ma gratitude va à ces témoins et à ceux qui m'ont aidée à les rencontrer. J'en profite ici pour remercier L. Niyonkuru, A. Hatungimana, P. Rwankara, A. Yengayenge et T. Nathan pour leurs précisions linguistiques et leur apport à ma compréhension des 
phénomènes de rumeur au Burundi, ainsi que V. Foucher et $T$. Dahou pour leur infinie patience et leurs commentaires roboratifs sur les ébauches de cet article.

8. Pour voter en 1960, les hommes devaient s'inscrire auprès des autorités administratives qui apposaient sur leur livret d'identité un cachet leur permettant de recevoir un bulletin le jour du scrutin (le cachet Gutora, qui confirmait qu'ils pouvaient " élire »). Une fois leur bulletin déposé dans l'urne, pour éviter qu'ils se présentent plusieurs fois et renouvellent leur vote, un second cachet était apposé sur leur livret individuel, portant la mention Yatoye (a voté).

9. À l'échelon administratif supérieur (province de Kayanza), le taux de participation moyen aux élections communales fut de $72 \%$, et à l'échelle nationale il atteignit $83 \%$. Dans les deux communes citées, la participation électorale s'est établie à $56 \%$ et $65 \%$ respectivement, et en outre on y compta un nombre de bulletins invalides (blancs ou nuls) bien supérieur à la normale.

10. Usumbura était le chef-lieu du territoire du Ruanda-Urundi, composé des « résidences » du Ruanda et de l'Urundi. Cette ville se situait dans la résidence de l'Urundi et il s'agit de l'actuelle Bujumbura, devenue capitale du Burundi à l'indépendance. Auparavant chacune des résidences avait son propre chef-lieu, à Kigali pour le Ruanda et Kitega (actuelle Gitega) pour l'Urundi.

11. Le lecteur aura compris que les termes " pédécistes » et « upronistes " ne sont pas des barbarismes mais des adjectivations courantes pour parler des membres et militants du PDC et de l'UPRONA.

12. Entretiens, $1^{\mathrm{er}}$ novembre 2004.

13. Entretiens, $1^{\text {er }}$ novembre 2004.

14. En 1960 déjà on notait que dans les régions où la rumeur circulait, les gens s'enfuyaient ou se cachaient à l'approche des véhicules (BUR 61/2: 28 novembre 1960). Mais après l'incident de Muzinda en 1961, la peur grandit et affecta plus massivement la population. Un témoin raconte que « beaucoup de gens cessèrent d'aller au marché par peur du cachet » et que " même à la messe ils craignaient d'aller communier »; partout, poursuit-il, « chacun regardait derrière lui constamment de peur qu'on ne lui appose le cachet » (Sakubu, 6 février 1990).

15. Construit à partir du nom du parti pro-hutu rwandais APROSOMA (Association pour la promotion sociale de la masse), ce surnom donné par les upronistes à leurs adversaires, avait une connotation péjorative marquée, puisqu'il faisait référence en même temps aux troubles de 1959 au Rwanda (la « Révolution sociale » hutue), à l'ethnicisation des rapports politiques dans ce pays, et à partir de 1961, aux républicains qui venaient d'y renverser la monarchie.

16. Voir aussi les articles consacrés à ces rumeurs de vaccinations dans les journaux coloniaux (BUR 60/2: 6 et 20 février, $1^{\mathrm{er}}$ mars 1960).

17. La piqûre est l'élément pivot de nombreuses rumeurs, pas seulement au Burundi, mais les interprétations qu'on peut en faire diffèrent selon les sociétés où elles se développent (MoRIN et al. 1982 : 42-43).

18. On fait référence ici à « l'idéo-logique » telle que l'envisage Marc Augé (1975), qui constitue une sorte de grammaire conceptuelle du monde, un répertoire de croyances et de présupposés qui construit la notion de l'individu dans un ensemble plus large de représentations sur la société et l'univers en général. Les actions et les réactions de chacun sont contraintes par les schémas d'interprétation imposés par cette logique. 19. Mes remerciements à Elias Sentamba pour ses éclairages à ce sujet. 
20. L'itinéraire politique et administratif de Rukuki peut être retracé grâce à de nombreux dossiers d'archives (cf. liste des sources).

21. Entretiens, 11 novembre 1990.

22. Le scrutin indirect au cours duquel Rukuki se fit élire député « intérimaire », en janvier 1961, avait installé une assemblée législative provisoire de majorité pédéciste et " démocrate », qui fut désavouée par l'upRonA. Les élections législatives de septembre 1961, organisées sous contrôle onusien, marquèrent la naissance d'une nouvelle assemblée, dominée par les upronistes, qui légiféra jusqu'en 1965.

23. Entretiens, $1^{\mathrm{er}}$ novembre 2004.

24. Son statut est confus et l'on n'a pas su s'il s'agissait d'un conseiller communal élu (mais son nom ne figure pas sur les listes officielles) ou d'un mushingantahe, l'un de ces « sages » conseillers que comptait chaque communauté de colline.

25. Entretiens, $1^{\mathrm{er}}$ novembre 2004.

26. Les recherches sur la mémoire nationale et son écriture montrent que la place respective des « rebelles » et des « fidèles » a bougé tout au long du $\mathrm{xx}^{\mathrm{e}}$ siècle, selon les évolutions politiques, les moments de rédaction et les sensibilités des lettrés burundais (C HRÉTIEN 1993b : 260-262, 1993c : 393-396).

27. Entretiens, $1^{\mathrm{er}}$ novembre 2004.

28. Ibid.

29. Une rumeur courante en 1960 racontait que l'impôt, individuel ou sur le bétail, n'était plus dû par les upronistes, ou pouvait être retardé, ou encore qu'ils « seraient exempts de tous travaux collectifs ». Dans les régions où elle circula, le recouvrement fiscal fut très imparfait cette année-là et le recrutement des travailleurs forcés plus difficile qu'à l'ordinaire (BUR 74/4 ; BUR 65/1 : 2 et 24 mai 1960).

30. Dans les enquêtes orales, les mots « propagande » et « rumeur » ont été utilisés de manière indifférenciée par les témoins pour parler de l'affaire du cachet. L'un de ces témoins a d'ailleurs raconté que « la propagande, c'était presque des sortes de rumeurs, chacun passait par ici, informait les siens, un autre faisait de même... Une fois renseignés, les gens se communiquaient l'information qui continuait à circuler de cette manière » (Ruduri, 11 octobre 1990).

31. Pierre Baranyanka était le patriarche de la lignée dynastique des Batare. Il était le père de J.-B. Ntidendereza, leader du PDC dont on a déjà parlé plus haut. Jean-Baptiste Ntakiyica était le cousin de ce dernier.

32. Officiellement, très peu de candidatures furent déposées en 1960 sous la bannière de l' UPRONA, mais en réalité la plupart des conseillers élus dans la commune de Muzinda et dans le « carré » des communes de Ngozi y étaient affiliés.

33. On ressent bien dans les enquêtes orales, notamment dans les entretiens menés à Muzinda, où il a été question des dizaines de personnes arrêtées à la suite du meurtre de Kayugi ( $1^{\mathrm{er}}$ novembre 2004), quelle a été l'importance pour la consolidation des adhésions upronistes de ce récit de l'oppression coloniale contre les nationalistes. 


\section{RÉSUMÉS}

$\mathrm{Au}$ Burundi, dans l'effervescence politique suscitée par la transition vers l'indépendance, les rumeurs ont constitué un support privilégié de l'information et de la propagande. À travers l'exemple de l'une de ces rumeurs, dite "du cachet », bien documentée par les sources orales et écrites, cet article propose d'explorer les imaginaires et les représentations populaires convoquées pour dire la politique dans le débat public que la décolonisation a ouvert au pluralisme partisan. Les déclinaisons chronologiques, territoriales et événementielles de cette rumeur permettent d'éclairer de manière originale les tensions et les enjeux politiques de la période. Non seulement elles mettent au jour les croyances de l'occulte et les ressorts de la fidélité monarchique qui dynamisaient alors la vie politique, mais encore elles dévoilent les processus différenciés de la structuration des audiences publiques et les recodages locaux de la réorganisation des pouvoirs qui ont accompagné les bouleversements politiques du début des années 1960.

The "Cachet" Rumor in Burundi (1960-1961): An Interpretation of a National Conversation on Politics. - In the agitation surrounding independence in Burundi, rumors were a favorite means for transmitting news and propaganda. The example of the rumor of the "cachet", well documented by both written and oral sources, is used to explore the folk imagery and ideas used to talk about politics. The chronological, territorial and event-related variants of this rumor shed new light on political tensions and issues at a time when decolonization was introducing multi-party politics. They expose driving forces in politics at the time (beliefs in the occult and the grounds of loyalty to the monarchy) and bring to light the differentiated processes of structuring audiences and locally recoding the reorganization of powers entailed by political upheaval in the early 1960s.

\section{INDEX}

Mots-clés : multipartisme, multiparty politics, sorcellerie, witchcraft, histoire politique, political history, décolonisation, imaginaire politique, monarchie, propagande et information, rumeurs, political imagery, monarchy, propaganda and news, rumors

Keywords : decolonization 